\title{
William Ritter et Edmond Bille : danse macabre et contredanse en temps de guerre
}

\author{
Philippe KaEneL \\ Université de Lausanne
}

\begin{abstract}
Durant la guerre, alors qu'il est de retour en Suisse, William Ritter fréquente le peintre neuchâtelois installé à Sierre, Edmond Bille. Leurs liens vont se tendre entre I9I7 et 1919, autour d'expositions en Suisse et en particulier à La Chaux-de-Fonds. Leurs complicités et leurs dissensions éclatent en 1919, alors que le peintre demande à l'écrivain de rédiger une préface pour son recueil intitulé Une Danse macabre.
\end{abstract}

Keywords: Edmond Bille, William Ritter, critique d'art, expositions, Première Guerre mondiale, Valais, danse macabre

La fréquentation d'Edmond Bille par William Ritter vers la fin de la Première Guerre mondiale paraît à la fois improbable et logique : improbable, au sens où les positions esthétiques et, plus globalement, idéologiques du peintre (de sympathie socialiste) et de l'écrivain (de sensibilité de droite), ainsi que leurs orientations religieuses et sexuelles ne sont guère au diapason ; logique, car leur expérience commune du canton de Neuchâtel, une certaine vision de la culture helvétique, un sens de la provocation, de l'indépendance et même un certain sentiment d'élection et de supériorité face à la médiocrité bourgeoise a pu les lier durant ces années de conflit. Tous deux sont des combatifs du crayon et de la plume, tous deux critiques d'art et artistes. Leur terrain de rencontre est alors le Valais et leur thème agonistique celui de la danse macabre. En même temps, l'un comme l'autre vivent dans ces années une série de tournants tant affectifs qu'économiques.

Voici esquissé le sous-texte de la rencontre de Ritter et de Bille, au cœur de l'entreprise macabre et du quotidien mortifère qui emporte le Continent et fait trébucher les équilibres culturels et linguistiques en Suisse. Mais qui sont alors les deux protagonistes de cette danse ou plutôt de cette contredanse ? On ne peut comprendre leurs mouvements et les figures qui en découlent sans se pencher plus en détail sur leurs parcours individuels et leurs positions entre I917 et 1919.

Edmond Bille naît en I878 d'un père instituteur devenu journaliste de sensibilité politique radicale, puis directeur d'école dans le canton de Neuchâteli. Formé à l'École des beaux-arts de Genève, à l'École nationale des arts décoratifs et à l'Académie Julian à Paris, puis à l'Académie de Florence,

I Sur Bille, voir notamment Wyder 2008 et le site web : www.edmond-bille.ch. 
il est en art un naturaliste tempéré d'un symboliste, comme tant d'autres artistes de sa génération. Influencé par le renouveau ruraliste qui anime les mêmes cercles, il fonde une colonie d'artistes à Brienzwiler dans l'Oberland bernois, en I897, et séjourne fréquemment en Valais, terre de prédilection des artistes primitivistes, dont certains comme Ernest Biéler ont été regroupés sous l'appellation d' «École de Savièse ".

Très tôt, il se distingue dans les arts graphiques, en particulier dans le domaine de l'affiche. Il se forme également en I899 dans l'atelier de Clement Heaton, réputé dans les arts décoratifs et actif à Neuchâtel. Un an plus tard, il découvre le val d'Anniviers en Valais et s'installe dans le village de Chandolin avant de retourner à Neuchâtel. En 1904, il épouse une femme issue d'une famille vaudoise aisée, Elisa Mayor. De suite, il acquiert à Sierre un terrain avec une sorte de château et des curies pour ses chevaux, auxquels il adjoint un atelier somptueux aux allures d'église gothique. En 1905, il édifie un grand chalet à Chandolin, puis achète l'année suivante l'ancienne propriété du grand peintre neuchâtelois, Maximilien de Meuron (I875-1968), au-dessus du Landeron, non loin du lac de Bienne. Du point de vue artistique, Bille gagne en notoriété sur le plan national à travers diverses expositions, concours et achats institutionnels. II expose souvent avec son collègue valaisan, Raphy Dallèves (I878-1940), en tandem au Helmhaus de Zurich en I9II et I9I6, et à la Galerie Bernheim-Jeune de Lausanne, en 19I7. Dallèves figure également présent, comme d'autres collègues, dans l'exposition que Ritter organise de concert avec Bille dans la Grande salle de l'Hôtel des Postes de La Chaux-de-Fonds, du I2 septembre au 9 octobre 19I8: Le Valais et ses peintres. Ritter s'est inclus dans les dix artistes sélectionnés et présente à cette occasion 38 aquarelles. Bille, qui s'attribue la place d'honneur, conçoit à cette occasion une affiche très atypique qui représente une Valaisanne en costume traditionnel, de dos, agenouillée devant un paysage alpestre et une nappe de brouillard d'où émerge une grande cheminée fumante (fig. I). Nous reviendrons sur cette exposition qui constitue l'un des moments significatifs de leur collaboration et de leur dispute.

Les thèmes de prédilection de Bille tournent alors autour de la peinture d'histoire (renouvelé par Ferdinand Hodler) et surtout de la ruralité valaisanne. En 1906, Ritter rend compte de l'œuvre de Bille dans la respectable Gazette des beaux-arts parisienne. Il le qualifie d'" espoir du groupe neuchâtelois, qui tout à coup écorche la langue de Segantini avec une telle âpreté » (Ritter I906: 262). Une œuvre importante caractérise ces années : La Mort et le bûcheron, grande toile panoramique datant de 1909, qui met en scène la camarde poussant (ou retenant) une luge dans un paysage de montagne enneigée, au crépuscule. Autre prélude à Une Danse macabre, il investit dans l'édition en finançant un somptueux ouvrage programmatique et auto-promotionnel, Le Village dans la montagne (Lausanne, Éditions 


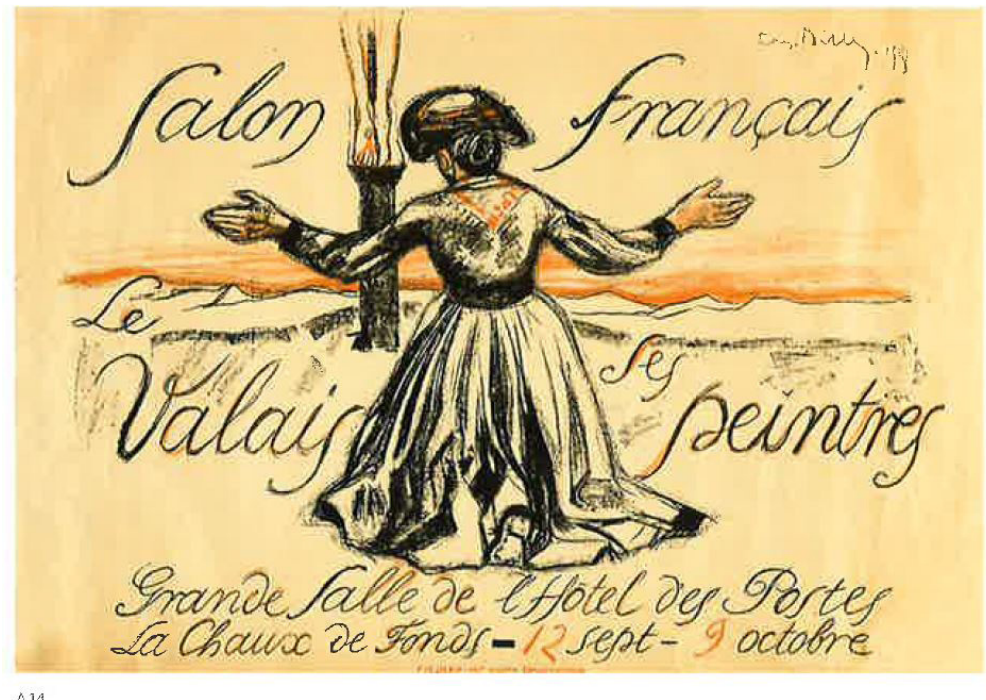

Fig. I : Salon francais / Le Valals ses peintres, 1918. Lithographie, 75 x $109 \mathrm{~cm}$ (extrait de Wyder, Edmond Bille, Estampes et affiches, 2014).

Payot, 1908), pour lequel il s'assure les services d'un jeune écrivain montant: Charles Ferdinand Ramuz.

En I9II, son épouse décède à l'âge de trente-quatre ans. La guerre éclate et le lieutenant Bille se retrouve mobilisé à la frontière jurassienne. Ses opinions politiques se radicalisent alors autour de plusieurs questions comme celle de la liberté d'expression menacée par la censure militaire, de la division entre Suisse francophone et germanophone, et de l'esprit germanique (prussien) qui envahit l'armée de milice helvétique. Peu après, il devient le chef de la place d'internement de Sierre, accueillant les soldats convalescents. Cette fonction le met en contact sur un autre plan avec la guerre et la question vivement débattue de la neutralité suisse. Cette question devient le thème d'un recueil de dessins satiriques, intitulé Au Pays de Tell (fig. 2). Financé par l'artiste et paru aux éditions Payot en 1915, le fascicule va connaître un certain succès commercial qu'attestent des cartes postales reprises de certains de ses dessins. Ces derniers fustigent la défaite morale de la Confédération, de cette "Suisse officielle » qui a décidé de fermer les portes à peine ouvertes de l'Exposition nationale suisse de Berne en I9I4. C'est dans ce contexte, à Sierre, que Bille fait la rencontre de l'écrivain Pierre Jean Jouve (I887-I976), avec lequel il s'associe pour un projet de danse macabre qui ne verra toutefois pas le jour (Martinet 1982 ; Kaenel 2003). Il fréquente plus largement les cercles pacifistes, réfugiés en Suisse, à Genève, dans lesquels figurent Romain Rolland (I966-I944), René Arcos (I88I-I959) 


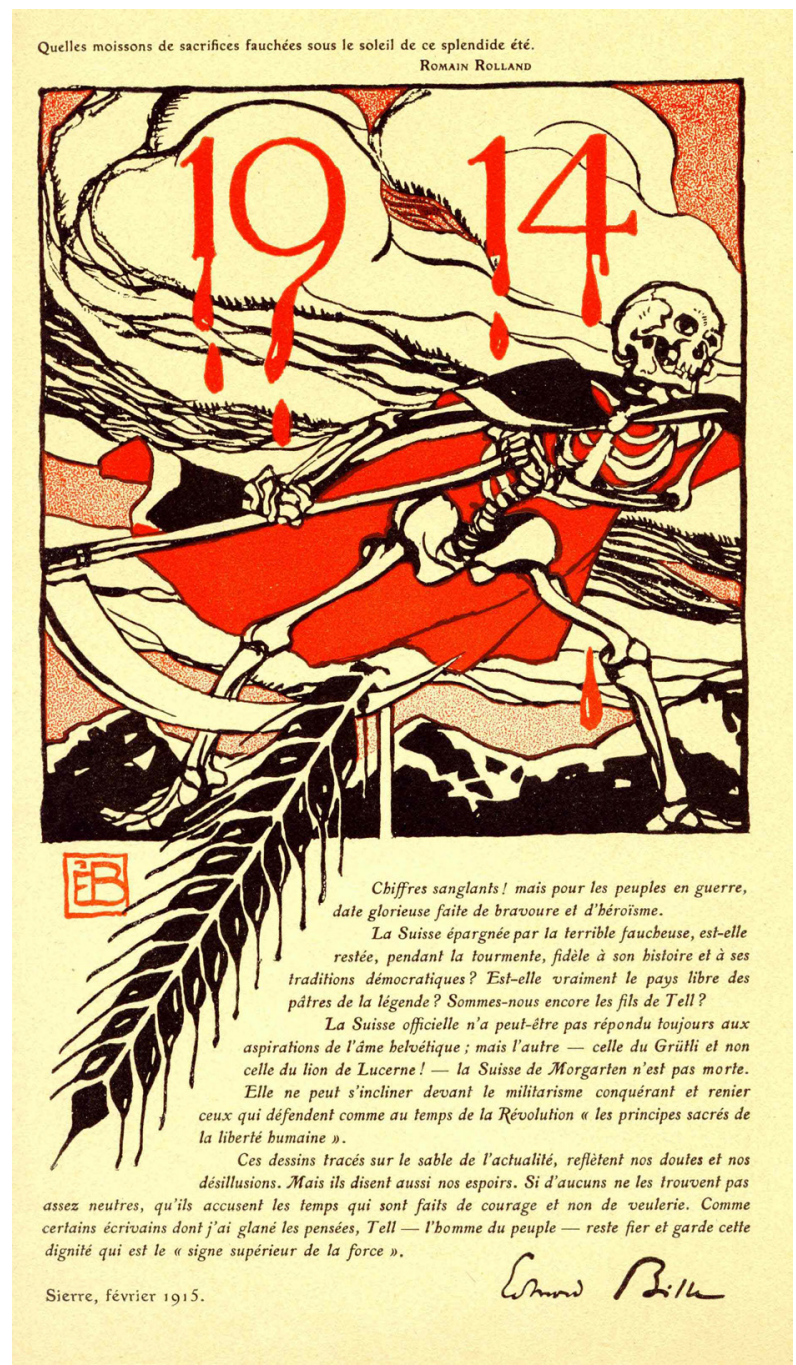

Fig. 2 : Edmond Bille, en-tête et préface, zincographie en rouge et en noir (extrait de: Au Pays de Tell, Lausanne, Payot, 1915. Lausanne, BCUL), photographie de l'auteur

ainsi que l'une des grandes figures à venir de l'imagerie politique, le Belge Franz Masereel (I889-1972), auteur de plusieurs danses macabres, dont l'une paraît de manière fragmentaire dans la revue genevoise Les Tablettes.

Tel est l'artiste que Ritter fréquente entre I917 et 1919. Bille est alors dans toute sa splendeur de châtelain socialiste, quelques années avant que la bourse ne s'effondre, en I92I, emportant une bonne part de sa fortune. Mais à l'époque, il peut se permettre de financer la publication d'une revue sati- 
rique de qualité, inspirée de modèles français et germaniques, L'Arbalète (I9161917). Jouve compose l'avertissement, signé par "La rédaction ", en tête du premier numéro, le Ir juillet I9I6 (le journal, publié à La Tribune de Lausanne, comptera en tout trente-quatre numéros et cesse de paraître à Noël 1917). Bille et ses partenaires principaux, les artistes vaudois Charles Clément (I889-I972), Victor Gottofrey alias Gotto (I89I-I920) et Maurice Hayward, alias Varé (1892-I95I), attaquent le manque de neutralité d'une classe politique jugé prussophile, les faiblesses de l'armée (et surtout de son général Ulrich Wille), les profiteurs de tous bords, ceux qui s'en prennent à la liberté d'expression, et de manière générale, tous ceux qui en Suisse ont contribué à la trahison des idéaux de la Suisse primitive. En cela, poursuivant en cela, L'Arbalète se place dans la droite ligne d'Au Pays de Tell.

Avec la cessation de parution du journal fin 1917, Bille revient en force vers la pratique de la peinture, mais publie à ses frais un dernier recueil qui flamboie d'une lumière noire, Une Danse macabre (fig. 3). C'est le recueil que

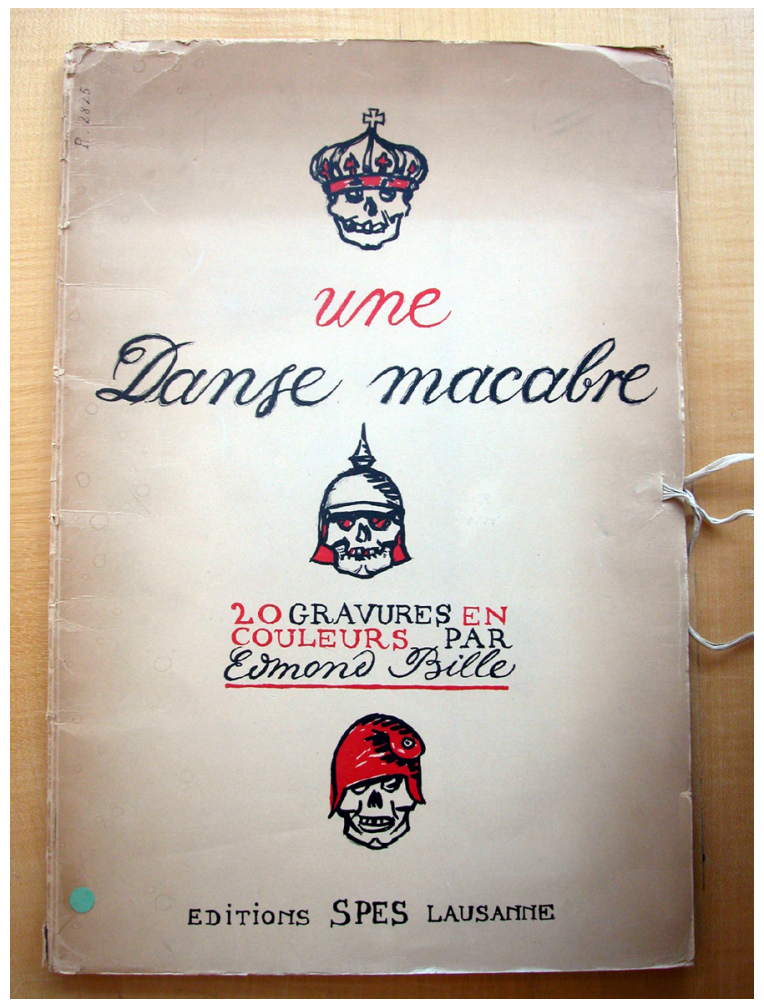

Fig. 3: Edmond Bille, vignette de titre, zincographie en noir et rouge, (extrait de : Une Danse macabre, Lausanne, Spes, 1919. Lausanne, BCUL), photographie de l'auteur 
Ritter va préfacer à la demande du peintre (fig. 4). Il est composé de vingt planches en chromotypogravure qui condamnent violemment à la guerre et vise toutes les cibles qui furent celles de ses deux publications antérieures. Il s'agit d'un projet certes militant, mais plus artistique, plus luxueux, qui prend position sur l'histoire du genre et l'histoire des arts en Suisse et en Europe. Ritter est plus que familier du thème, mais il ne le perçoit pas de la même manière, à tel point que Bille va demander une autre préface pour l'édition courante de son recueil. Pour ce faire, il mandate le journaliste William Matthey-Claudet (I882-I952), un Jurassien natif de Môtier-Travers, qui avait publié dans Les Tendances nouvelles un article richement illustré sur l'œuvre de Bille à l'occasion de l'exposition parisienne annuelle de l'Union internationale des Beaux-arts et des Lettres, en 1912. Trois ans plus tard, il réitérait l'hommage dans la revue genevoise Pages d'art, enrichi d'une quinzaine de pages et 44 illustrations.

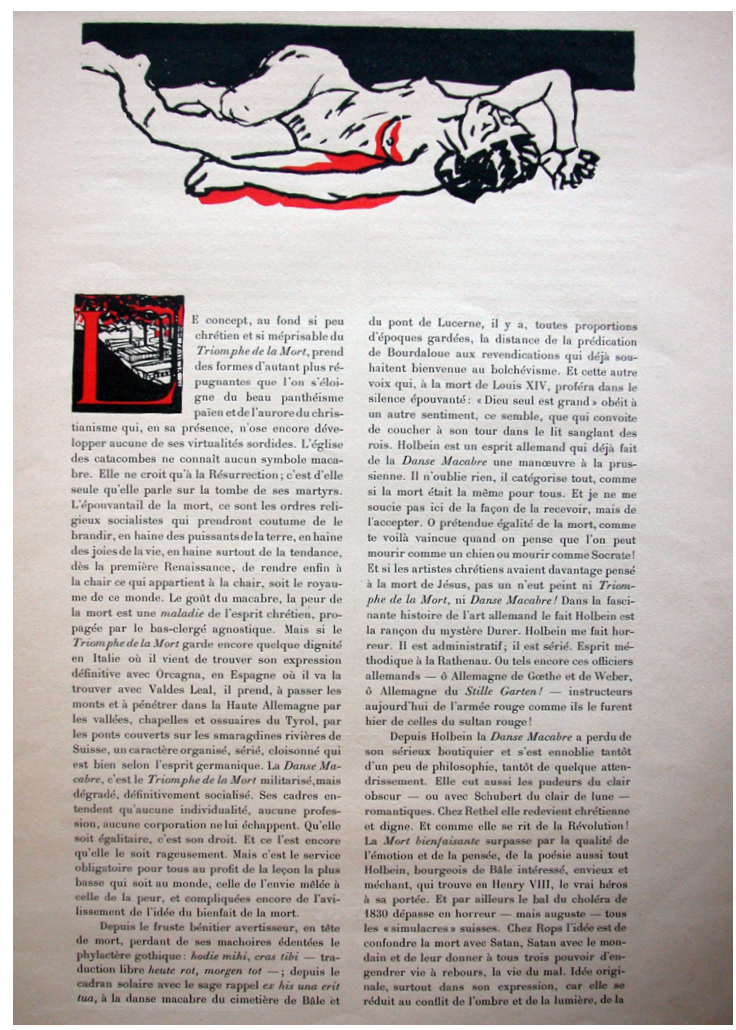

Fig. 4 : Edmond Bille, Préface et lettrine, zincographie en noir et rouge, (extrait de: Une Danse macabre, Lausanne, Spes, 1919. Lausanne, BCUL), photographie de l'auteur 
Pour comprendre les raisons de la dispute autour d'Une Danse macabre, il faut prendre conscience de la situation spécifique de Ritter à la fin de la guerre. Le récit des interactions entre l'artiste et l'écrivain resurgit dans strates écrites de profondeurs diverses, entre déclarations publiques et témoignages personnels, entre des textes publiés, correspondance et journal intime.

\section{Récit d'une guerre}

À l'aube du conflit armé, Ritter doit rentrer en Suisse après quatorze années passées à Munich, dont une partie avec son nouveau compagnon, Janko Cádra, avec lequel il a parcouru l'Europe, de Prague à Aix-en-Provence en passant par Neuchâtel. Dans ces années, Ritter s'investit plus fortement dans la pratique du dessin et de la gravure. Installé au Landeron, petite cité médiévale non loin du lac de Bienne (là où Bille a acquis une demeure), il déménage dans le Jura, non loin du Doubs et de la frontière française, à La Rançonnière, aux Brenets. Cette demeure sera son port d'attache jusqu'en 1922. Il y réside également avec un beau jeune homme, Henry, apprenti mécanicien, qui lui sert notamment de modèle, et qui va rompre en I9I8 sous la pression de ses parents désireux de le marier alors qu'il a atteint sa majorité. Ritter en sera très affecté alors même qu'il rompt, sur un autre plan, avec Bille.

En I9I7, les liens de l'écrivain avec Bille et le Valais se renforcent au fil de trois séjours, dont le premier de quatre mois à Varone où il prépare entre autres des conférences publiques sur l'histoire et la culture du canton. Ritter a fait le récit de ce morceau vie dans un manuscrit autographe intitulé « Mes relations avec les artistes suisses » :

La première fois, il s'agissait de fournir à une publication d'étrennes suisses un article sur Edmond Bille (Ritter [1918]). La seconde fut un long séjour d'automne 1917 et d'hiver et de printemps jusqu'à fin mai 1918, à Varone entre Sierre et Loèche-la-Ville. Après quoi nous organisâmes à La Chauxde-Fonds une imposante exposition des artistes valaisans. Et l'automne et l'hiver suivants nous les passâmes [...] à Borsuat [Borzuat] sur Sierre.

Ritter mentionne incidemment la visite d'artistes neuchâtelois comme la peintre Blanche Berthoud (I864-I938), fille peintre neuchâtelois Auguste-Henri Berthoud, et du Loclois Maurice Mathey (I878-1975), avant de glisser : « Il n'y a pas eu à proprement parler d'artistes valaisans avant que des Genevois et des Vaudois s'avisassent de demeurer et pour ainsi dire de s'approprier le Valais ». Tel est le cas des peintres Édouard Ravel, Ernest Bié- 
ler et Edmond Bille, ce «vrai caméléon ». Il poursuit en brossant un portrait caustique de ce dernier :

Avant tout M. Bille entend jouir de la vie d'une façon plénière. La peinture n'arrive que comme adjuvant à des succès éphémères de vanité, de plaisir, de gloriole au jour le jour. Faire parler de soi, bien! Mais laisser quelque grande œuvre et si possible un ou deux chefs-d'œuvre, l'artiste était assez bien doué pour que nous ayons pu attendre de lui pas assez pour résister aux tentations de la facilité et aux séductions et dissipations de la vie trop aisée. On a toujours l'impression avec lui que le travail est une distraction de plus et qu'il ne saurait avoir d'autre but que de lui rapporter des satisfactions de vanité. C'est ce qu'il appelle être optimiste. Il sait être drôle. Par des feintes de franchise audacieusement cyniques. Une façon d'avoir l'air de se livrer, de convenir qu'il faille le prendre comme il est. Au demeurant, bien décidé de se servir de tout et de chacun aux seules fin de servir aux amusements et aux intérêts d'Edmond Bille. Caractéristiques : il s'est toujours brouillé avec tout un chacun. Quitte à se réconcilier parfois. Quand cela s'avérait décidemment utile (« Mes relations avec les artistes suisses »).

Suivent des lignes féroces sur les ambiguïtés d'un personnage alternant fonctions publiques et activité de pamphlétaire "bolchévique "; sa familiarité et son usage des élites politiques comme le conseiller d'État devenu conseiller fédéral, Robert Comtesse ; son « étrange bicoque, toute faite de pièces rapportées, un castel d'exposition vieux jeu », à Sierre. Surtout, Ritter en veut à Bille d'avoir pu imaginer instrumenter sa critique :

Ce fut aussi chez lui que je rencontrai pour la première fois une façon dont je devais plus tard me convaincre qu'elle doit être assez endémique à la Suisse romande de prétendre s'assujettir un critique attitré, à le réduire en quelque sorte à ne dire ou n'écrire que ce qui convenait à l'artiste et comme il lui convenait. Il va sans dire que si l'on avait spéculé sur ce genre de servitude on s'était bien trompé. Je me hâte d'ajouter que M. Bille était au reste assez avisé pour ne pas insister dès qu'il reconnaissait avoir fait fausse route («Mes relations avec les artistes suisses »).

Le récit devient ensuite plus anecdotique, notamment sur la « paillardise » de ce « débauché » qui trompe sa seconde femme (que Ritter apprécie grandement) avec une jeune fille de Sierre, Berthe, sur ses « airs de grand nabab ", " volage ». Et de condamner les « fantaisies délurées auxquelles sait se complaire ce peintre religieux qui ne croit pas à grand-chose et qui a su enjôler le clergé des villages valaisans où il lui a plu de badigeonner quelques murs d'église propices ». Ritter poursuit avec la démolition en règle de sa « peinture de gros faiseur » et une vision prospective : 
L'histoire de l'art suisse aura vite fait de tourner la page, toute divertissante que saurait être la biographie. Dieu m'en préserve de l'essayer jamais ! Toutefois si peut-être, quelque jour, ceci peut server à en documenter quelque peu le chapitre de ces années de guerre, voici. On ne doit aux morts que la vérité et M. Bille est mort pour moi. Comme pour tant d'autres ! (« Mes relations avec les artistes suisses »).

Le violent ressentiment de Ritter envers Bille mêle divers niveaux : professionnel (la défense de son indépendance critique) ; économique (les difficultés vécues par Ritter qui s'efforce de reconstituer et rentabiliser ses réseaux en Suisse) ; idéologique (son idéal aristocratique l'incite à mépriser toute forme d'arrivisme) ; politique (Ritter qualifie Bille de bolchévique et d'anarchiste) ; mais aussi et surtout moral car, bien qu'homosexuel sautant de jeune en jeune, l'écrivain, au nom de valeurs « bourgeoises » et conservatrices, condamne sans appel les infidélités maritales de l'artiste ainsi que sa duplicité arrogante.

Reprenons ici le récit de leurs relations en suivant la chronologie, sur la base de la correspondance et du journal intime qui donnent une autre couleur à leurs relations. Le 25 août I9I7, Bille écrit à Ritter:

J'ai lu avec intérêt votre étude sur l'exposition de Zurich dans la Semaine littéraire $^{2}$. Vous êtes un des rares écrivains, peut-être le seul, qui ait su faire de la critique d'art une chose vivante et colorée ; je vous lis toujours avec passion, même quand vous êtes si injuste, même quand vous dites tout le contraire de ce que je pense. Ainsi, je ne pense pas un mot de ce que vous écrivez sur le nommé Cingria ; dans notre art suisse actuel - et qui sait s'il ne peut devenir l'embryon d'un art européen - ce faux Genevois, ce Dalmate, ce précieux, ce snob, est un anachronisme ; que dis-je un champignon vénéneux [...] votre âme de néo-slave impénitente vous fait voir des richesses où il n'y a que des pauvretés »(Correspondance).

Le ton est donné : l'artiste ne partage pas les positions de l'écrivain mais admire en lui un esprit d'indépendance et même de provocation dans lequel il se reconnaît. Leurs échanges vont ensuite porter sur les expositions de Bille à Zurich au printemps 19ı18, Gemälde-Ausstellung, Walliser Maler. Edmond Bille, Raphy Dallèves, Henry van Muyden im Helmhaus Zürich, (I6.3 - 2.4.I9I8), soutenues par une salve d'articles de Ritter qui accompagnent les expositions de Bille en Suisse en I9I8. Ainsi, le I2 mars, le peintre demande au critique des changements dans l'article sur son exposition:

2 Bille s'était déjà exprimé sur la critique de Ritter dans son article violemment antihodlérien : «L'art suisse au pilori », La Semaine littéraire, [juillet] I9I3 [?]. "À propos d'un article de William Ritter "La Suisse à l'exposition de Munich" ", La Semaine littéraire, 28 juin I9I3, pp. 309-3II. 
" "couillonner" ne va pas. C'est vulgaire et personne ne le comprendra [...] autre chose, mais pas choléra (ni "colique ", pour le ton de ma Valaisanne à l'escalier. [...] Le reste parfait, sans retrancher une ligne ou un mot. Et merci - c'est extrêmement vivant, drôle, ... et souvent vrai »(Correspondance).

L'article paraît peu après (Ritter 1918) et Bille s'en déclare ravi le 2 avril : " vous avez dit là des choses qui me sont précieuses, et qui pour moi, devraient être dites surtout en ces temps où la lutte n'est plus contre les œuvres, mais contre les hommes! Rien que pour votre article, j'aurais fait mon exposition » (Correspondance). On y lit quelques phrases sur «ce succédané suisse à la façon dont il est, en sa vie et ses allures, du seigneur féodal que je viens de dire. Selon moi qui l'écris, c'est un grand éloge. Pour faire penser à de grandes choses il faut déjà que les choses soient grandes. Il est certain que M. Bille est ce que le canton de Neuchâtel, par le moyen du Valais, a apporté de mieux à l'art suisse ces vingt dernières années » (Ritter I9I8: 572).

Constatant l'échec de cette dernière exposition le 26 mars, Bille fustige le "Boykott suisse ", accuse le galeriste zurichois (Marküs), ses prix, tout en reconnaissant que son changement de style a pu surprendre, et sa réputation de pamphlétaire le dévaloriser (« on boude en moi le dessinateur de Tell et surtout de L'Arbalète»). Et de conclure dans un vocabulaire caractéristique des tensions contemporaines : "J'ai eu de tels écœurements en voyant ce marché bocho-pictural et ses procédés judaïco-artistiques que j’aimerais être mis pour toujours au loin de ces entreprises [...] » (Correspondance).

D'autres affinités entre l'artiste et l'écrivain apparaissent au fil de leurs relations, parmi lesquelles leur détestation commune de la critique d'art du professeur Philippe Godet (I850-I922), une autorité neuchâteloise, correspondant à la Gazette de Lausanne, avec lequel Ritter avait eu l'occasion de croiser la plume à maintes reprises autour de 1900 (Kaenel 1993). Ainsi Bille déclare-t-il le 25 avril I9I8, ne pas vouloir répondre à un article de Godet : "C'est du temps perdu, les imbéciles sont de tous les mondes, et le spirituel Ph. Godet me paraît mûr pour l'hospice!». Ritter ne pouvait qu'approuver.

La correspondance adressée à l'écrivain évolue de "cher Monsieur» à «cher ami Ritter » durant l'été I9I8. À l'époque, tous deux travaillent à l'exposition Le Valais et ses peintres de La Chaux-de-Fonds. Organisateur, critique et exposant, Ritter dresse son autoportrait biographique dans le catalogue où il justifie sa présence en tant qu'exposant : « N'a jamais cessé de peindre et dessiner en marge de ses voyages, de ses travaux d'écrivain et des émotions capitales de sa vie qu'il doit à la musique. [...] Est allé en Valais par hasard, mais avec un vague espoir d'y trouver une petite compensation à tant de paradis, perdus par les frontières fermées, et il y a travaillé comme partout selon son cœur et les choses que disent à son cœur les sols qu'il foule »(Ritter I9I8). 
Rapidement, les susceptibilités et dissensions s'installent. Le I5 juillet, Bille déclare au critique : « Je reconnais tous vos droits sur l'exposition, et serai votre très obligé - soyez sûr que je ne ferai jamais rien en sous-main » (Correspondance). Leur litige porte entre autres sur l'affiche, comme le révèle une lettre de Bille le 20 juillet à propos du galeriste Bendlé : «Je lui ai dit mon étonnement de ne pas voir figurer parmi les «Valaisans » Virchaux [le Loclois Henri Édouard Huguenin-Virchaux (1878-I958)] et Albert Muret [Vaudois (I874-I955) qui s'est construit un chalet à Lens près de Montana]. Cela ressemble à une exclusion - Je lui ai conseillé de scinder - au catalogue - les deux groupes : a/ les Valaisans b/ les Neuchâtelois ayant peint le Valais (y compris Matthey du Locle !). Cela me paraît nécessaire pour expliquer l'abstention des "Genevois en vacances »! - et des autres - Et voilà tout; ne m'accusez de rien d'autre» (Correspondance).

Durant la même période, les relations entre Bille et Ritter deviennent intimes sur un autre plan. Le peintre se confie sans détours au critique qui connaît bien sa maîtresse, Berthe : «Elle m'a plaqué pour coucher depuis longtemps avec des internés ("alliés" du reste ! et je ne m'en doutais pas) ", lui écrit Bille le 30 avril. Quelques mois plus tard, le 3 septembre, il annonce qu'elle est revenue, repentie : ce n'est plus la « fillette » mais « c'est la femme qui devient "tortionnaire" ». À la fin de l'année, Bille ira jusqu'à solliciter la complicité de Ritter : «Bille de nouveau chez nous ce matin. Il voudrait que nous fissions semblant de continuer de louer ceci après notre départ ; c'est pour y installer sa Berthe! Moi je me fais des scrupules à l'égard de Mme Bille. Tremper dans une aussi sotte histoire! Par ailleurs il nous a apporté la nouvelle du coup d'état bolchéviste de Berlin dont il jubile...» (Journal intime, 29 décembre 19I8). En même temps, sur le plan sentimental, Ritter vit fort mal l'attitude de Bille face à ses compagnons masculins. Dans son journal intime en date du I2 septembre, à l'occasion du vernissage de l'exposition de La Chaux-de-Fonds qui réunit le ménage à trois des Brenets, Ritter fulmine : "Bille nous invite à déjeuner mais ne se tient pas de faire sentir à Jean [Janko] et surtout à mon pauvre Henry combien il leur en veut d'être obligé de les tolérer. Après sa conduite à leur égard pendant ce repas je l'étranglerais bien ».

Sa rancœur est également d'ordre financier, comme nous l'apprend son récit rétrospectif, «Mes relations avec les artistes suisses » : " À l'exposition valaisanne, qui ne me rapporta absolument rien en dehors du prix que me fut payé le catalogue, ce qui fut loin de couvrir mes frais de correspondance avec les artistes, mes déplacements des Brenets à La Chaux-de-Fonds, et à plus forte raison mon temps, mes articles - pas une ligne de l'ensemble de ce travail qui ne fut dactylographiée à mes frais, - il va sans dire qu'Edmond Bille occupait la place d'honneur et qu'il lui fut réservé la part du lion dans 
la surface à couvrir». N'oublions pas que Ritter faisait partie des artistes exposants...

L'humeur de l'écrivain face à l'artiste oscille considérablement à cette époque. «Bille est venu pour la soirée chez nous ; ça c'est gentil ! [...] Et la causerie fut charmante ", indique le journal intime le 2I novembre I9I8. Quelques jours plus tard, par contre, il s'irrite de sa venue :

[...] au moment de commencer mon aquarelle, Bille est arrivé prendre le café avec nous et s'est éternisé. Il a essayé de m'intéresser à son ami Glor [Gloor], incarcéré pour excitation antimilitariste ${ }^{3}$. Quel désordre dans cet esprit de Bille! Sauf jouir de la vie, d'avoir du succès, d'épanouir sa grosse personnalité, il n'est que contradiction et anarchie. Aucun sens du devoir, d'un devoir quelconque ! Et une crainte de la mort ! En l'espèce de la grippe qui vient de leur prendre quelques connaissances » (Journal intime, 29 novembre 1918).

Cette crainte de l'épidémie et le contexte obsessionnellement mortifère de la guerre incitent Bille à développer dans un recueil de luxe un thème qu'il a traité plusieurs fois en peinture comme en gravure : celui de la danse macabre. Bille se tourne vers Ritter une fois de plus : « Hier soir chez Bille décidé que je ferai la préface de sa Danse des Morts ", relève-t-il dans son journal intime, le I7 janvier I9I9. Quelques jours plus tard, le 23 mars, l'artiste envoie des précisions à l'écrivain (fig. 5) :

Pour vous ? Bâtir un texte; nous disposons comme place de I6o lignes de 45 lettres. [...] Ne pensez-vous pas qu'il serait intéressant de rappeler que le thème « Danse macabre » est un vieux thème suisse, / Holbein, Manuel, etc. / Et que je reste dans la tradition en le reprenant. Mais ma mort est ici le vice social qui engendre la guerre, et ses suites »(Correspondance).

Le soutènement socialiste de cette danse macabre n'est pas sans irriter Ritter qui ne cache pas ses sympathies monarchistes : «Il parait que Bille navigue ces temps avec Glor [Gloor], sorti de prison où il avait été mis pour excitation de soldats à la désertion lors de la guerre. C'est toujours le système des fréquentations compromettantes. Et cette Danse macabre qu'il faut préfacer s'en prend aux souverains, aux diplomates et aux capitalistes en se gardant bien de toucher au bolchévisme » (Journal intime, 27 mars I919). Au fil des jours, Bille ne cesse de lui demander des modifications : «J'ai trouvé à sa préface de petites améliorations cette nuit et lui redemande le premier feuillet. Lui de son côté me demande encore des corrections, toutes les cor-

3 Ernest Gloor (I893-1964) a présidé les Jeunesses socialistes dès I9I6. Le I8 décembre I9I8, il a été condamné par le Tribunal militaire territorial à trois mois de prison suite à une dénonciation l'accusant d'avoir essayé de détourner des soldats de leur devoir (http://www.davel.vd.ch/ qfpdavel/333/Fi66752I.pdf). 


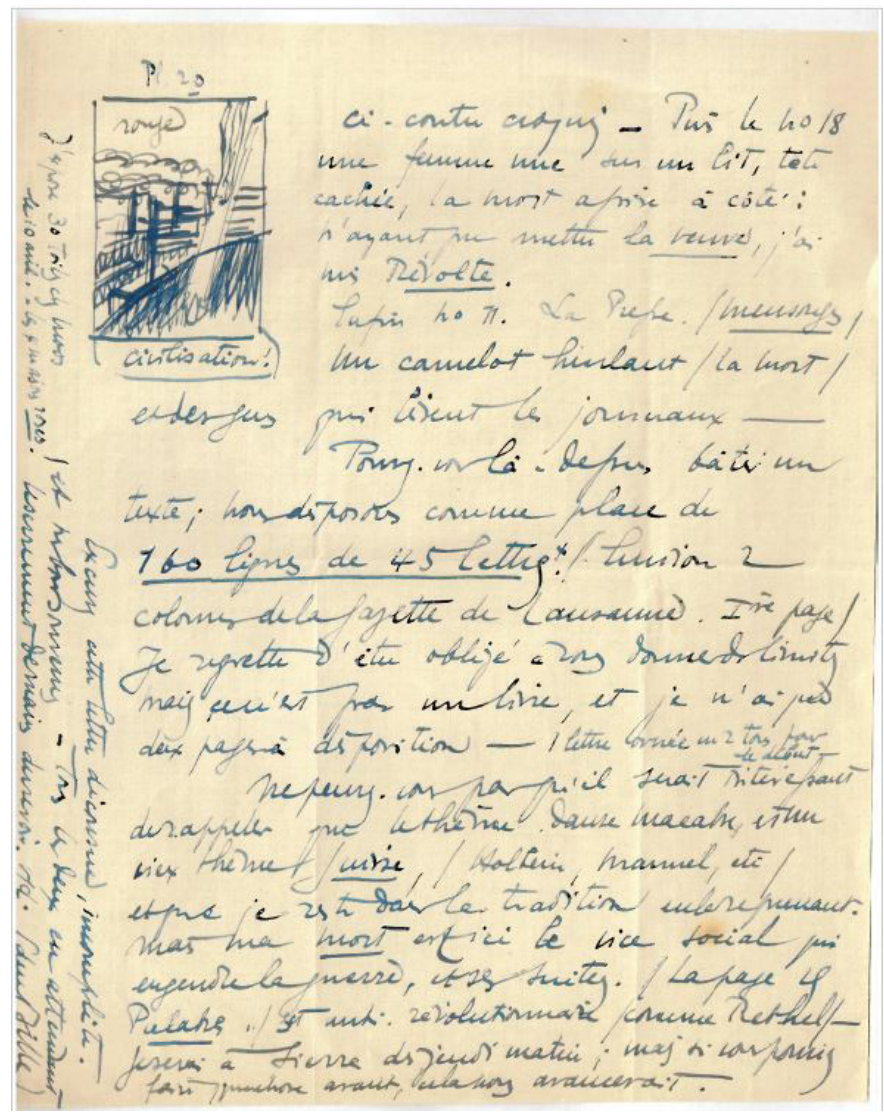

Fig. 5 : Lettre illustrée d'Edmond Bille à William Ritter, le 23 mars 1919, Berne, ALS, Fonds William Ritter, Correspondance.

rections de la couardise et de la basse vanité [...] (Journal intime, 3I mars 1919). Quelques jours plus tard, Ritter surenchérit : «Sa Majesté Bille nous a fait prier de passer chez lui prendre le café à une heure et demie. Sa Majesté a une lettre de l'éditeur à me communiquer à propos de la préface [...]. Mais si on me chicane trop avec la préface je la retire. Et c'est la première chose que je dis à Mme Bille lorsqu'on nous fait attendre dans la bibliothèque où l'on nous a fait entrer. Ma fébrilité est d'ailleurs si évidente que Bille coule tout doux » (Journal intime, 2 avril I9I9 ). La tension apparaît clairement dans la lettre que Bille écrit le I8 avril, après réception de la version définitive de la préface : 
Cher ami Ritter,

Merci pour votre article que je vous renvoie. C'est ce qui s'appelle recevoir une « volée de bois vert ». Mérité ou non, le geste ne me déplaît pas ; je ne vous reproche qu'une chose ; c'est d'avoir pris trop de précautions pour qu'on ne s'aperçoive pas que cette "danse ", somme toute, vous embête, tandis qu'au fond vous en pensez beaucoup de mal.

Mais pour qui sait lire, on ne saurait s'y tromper ; je souhaite à vos lecteurs, dans ce domaine, un flair égal au mien - du moins votre écrit ne sent-il pas le « copain » - je pense à tant d'autres qui à votre place distribuent sans compter leur eau bénite de cour - vous avez raison de garder la vôtre pour ceux qui en sont dignes.

Résumé : votre article me déplaît, mais je ne vous en veux pas le moins du monde, et je reste votre amicalement dévoué Edm. Bille.

Dans ses souvenirs manuscrits, Ritter a fait le récit de ce désaccord virant à la dispute :

[...] le séjour de Varone avait abouti à divers articles, et à l'exposition de La Chaux-de-Fonds, celui de Sierre devait être marqué par la préface à la Danse Macabre qui succédait aux préoccupations de l'Arbalète. Encore fallut-il, bien que l'on m'avait assuré une pleine liberté, passer par toutes les exigences de détail de l'intéressé. J'y consentis sans difficulté : N'était-il pas chez lui puisqu'il assumait tous les frais d'une publication luxueuse qui, de nouveau, n'était pour lui qu'une affaire de vanité. Encore eûs-je tôt fait de me rendre compte qu'il n'y avait pas eu assez de fanfare dans mon texte. Allons ! on se séparerait sans doute aussi peu satisfait les uns des autres que selon la coutume de toutes les collaborations de M. Bille avec des tiers (" Mes relations avec les artistes suisses»).

Ritter ne soumet évidemment pas à ce régime de "vassalité complète " (tels sont ses termes) qui caractérise à ses yeux William Matthey-Claudet, l'auteur de la seconde préface à Une Danse macabre.

Ce litige fait resurgir la dimension financière larvée de leur relation, Bille ayant prêté à Ritter sept cents francs le 29 mai I9I8, et lui les réclamant alors. L'écrivain - qui est dans l'attente des revenus issus de la vente des collections de son père et de ses terrains - y voit une manière de l'artiste pour faire pression sur son indépendance et le contenu de sa préface. L'affaire est à tel point vive dans la mémoire de Ritter qu'il rend compte de leur dernière rencontre explosive sous la forme d'un dialogue dans « Mes relations avec les artistes suisses ». Une dernière lettre de Bille à Ritter, le 6 mai I9I9, révèle ce tissu de malentendus personnels et financiers, sous la forme d'une dénégation : «J'ai cru bien faire en ne vous en parlant pas jusqu'ici - ce qui ne veut pas dire que j'aie beaucoup apprécié ce silence ; aujourd'hui, - un an après - les circonstances m'obligent à le rompre ; d'autre part, votre attitude à mon égard - je dis votre attitude, prière de ne pas faire de rapprochement 
avec votre article ! - n'est pas faite pour m'engager à user vis-à-vis de vous avec beaucoup de ménagement »(Correspondance).

\section{Contredanses autour de Holbein}

Une Danse macabre a fait l'objet de deux éditions. La première, de luxe, à vingt exemplaires sur papier Hollande au prix de cent francs (plus cinq pour l'auteur) ne fut pas commercialisée. C'est justement la version qui comporte la préface de Ritter. La seconde édition comptait cinq cents exemplaires vendus vingt-cing francs et comportait des petits textes de Bille expliquant les images. Ces exemplaires étaient introduits par la préface de William Matthey-Claudet. Mais revenons au texte même qui débute en ces termes :

Le concept au fond si peu chrétien et si méprisable du «Triomphe de la Mort " prend des formes d'autant plus répugnantes que l'on s'éloigne du beau panthéisme païen et de l'aurore du christianisme qui, en sa présence, n'ose encore développer aucune de ses virtualités sordides [...]. L'épouvantail de la mort, ce sont les ordres religieux socialistes qui prendront coutume de le brandir, en haine des puissants de la terre, en haine des joies de la vie [...]. La « Danse macabre », c'est le « Triomphe de la Mort » militarisé, mais dégradé, définitivement socialisé (Ritter 1919).

Voici qui n'était guère pour plaire à la sensibilité politique de Bille ! Ritter poursuit en associant les décors macabres du cimetière de Bâle et du pont de Lucerne à l'esprit du « bolchévisme».

Holbein est un esprit allemand qui a déjà fait de la «Danse Macabre » une manœuvre à la prussienne. Il n'oublie rien, il catégorise tout, comme si la mort était la même pour tous. [...] Dans la fascinante histoire de l'art allemand, le fait Holbein et la rançon du mystère de Dürer. Holbein me fait horreur. Il est administratif ; il est sérié. Esprit méthodique à la Rathenau. Ou tels encore ces officiers allemands - ô Allemagne de Goethe et de Weber, ô Allemagne de «Stille Garten »! - instructeurs d'aujourd'hui de l'armée rouge comme ils le furent hier de celles du sultan rouge!

Depuis Holbein la « Danse Macabre » a perdu de son sérieux de boutiquier et s'est ennoblie tantôt d'un peu de philosophie, tantôt de quelque attendrissement. [...] Chez Rethel elle redevient chrétienne et digne. Et comme elle se rit de la Révolution ! ( Ritter I9I9).

Son attaque de Holbein qui ne lui sera pardonnée ni par Bille, ni par les critiques contemporains. L'écrivain genevois Robert de Traz (I884-I95I), grand défenseur d'une spécificité de la littérature suisse, cofondateur de la Nouvelle Société Helvétique (I9I2), tient une chronique dans La Semaine lit- 
téraire où il jette : «M. William Ritter écrit en tête de l'ouvrage une préface dont quelques termes sont imprévus. Holbein lui fait « horreur » : c'est peutêtre réciproque [...]. M. Ritter n'a pourtant plus l'âge où l'on s'imagine épater son lecteur en vilipendant un grand artiste » (Traz I9I9). Dans une ligne analogue, Romain Roland glisse en passant à l'artiste qui lui a envoyé son recueil de planches :

Mais vous méritez une autre préface. J'ai autrefois été attiré par l'intelligence curieuse et ondoyante de W. R. Mais j'ai été déçu. Il y a, au fond, beaucoup d'étroitesse dogmatique et pas mal d'ignorance. N'insistons pas sur la fessée qu'il inflige à Holbein! (C'est tout de même un peu cocasse.) Mais il est bien bon d'attribuer aux boches l'invention de la « Danse macabre ", «ce triomphe de la mort militarisé »! Il devait savoir que c'est au cimetière des Innocents en plein Paris de Charles VI, que cette « Danse » a jailli et s'est épanouie sur la peste et le plaisir (Martinet I982 : I43).

La réaction nationaliste de l'écrivain français est à l'unisson de la vision qu'avait l'artiste de son œuvre qui avait pour but de renationaliser le genre, mais du point de vue suisse : "Le thème était bien de chez nous : je renouvellerais la tradition »(Bille I939 : I67). De ce point de vue, Une Danse macabre se place dans le prolongement d'une guerre idéologique portée par les images. La position de Ritter envers la culture allemande entre évidemment en conflit avec ce projet.

De manière subtile (pour ne pas dire insidieuse), tout en critiquant l'Allemagne militarisée, Ritter s'en prend aux modèles auxquels Bille rend précisément hommage (Holbein et Rethel) en allant jusqu'à les pasticher, sur fond de condamnation de l'esprit bourgeois et du socialisme.

Et voici M. Edmond Bille ! [...] M. Bille s'en tient à la donnée internationale et un peu socialiste. Et un peu simpliste aussi : peuple, on te trompe ; soldats, on abuse de toi ; ouvrier, on t'exploite ; journalistes, vous mentez ! Eh ! je n'en disconviens pas. Mais que fait-on donc en Russie sous prétexte d'avoir corrigé tout cela ? Et alors pourquoi deux poids, deux mesures ? Pourquoi reconnaissons-nous ici quelque peu François Joseph et pas le moindre Lénine ? Et pourquoi la haine écume-t-elle les seules tranchées - où c'est la guerre - et pas les prisons de Petrograd - où ce devrait être l'Eldorado? (Ritter 19I9)

Non content d'ironiser autour du projet culturel et idéologique de Bille, Ritter, en conclusion, envoie valser les fondements de la démocratie helvétique dont l'artiste ne critique que les dérives contemporaines. La préface se clôt sur ces lignes désabusées et elliptiques : 
Une fois de plus, la constatation désenchantée restera cruellement vraie : on ne fait que changer de victimes. Justice égale pour tous est un vain mot, et « la plus ancienne démocratie du monde » met à nous le prouver ces temps un entrain que nous n'attendions pas de ses cheveux blancs. Il est vrai que d'aucuns affirment qu'elle soit chauve... (Ritter I9I9).

En d'autres termes, le texte de Ritter sur Une Danse macabre tient à la fois de la contredanse, de la pirouette et du retournement de sens, tandis que la préface de William Matthey-Claudet inverse le mouvement. Elle replace les origines du genre en France (comme le voulait Romain Rolland), établit les filiations suisses du genre, de Bâle à Neuchâtel en passant par Lucerne, avant de revenir au commentaire critique de Rethel sur les journées révolutionnaires de juin I848. Et l'auteur de conclure : "L'œuvre de M. Bille, toute frémissante d'indignation et de pitié, donne à penser. Elle est puissante, âpre et brève, sans ornements romantiques, et restera comme un témoignage fidèle des jours abominables que nous venons de traverser ».

Un autre sous-texte alimente le malentendu logique qui tend les relations entre Bille et Ritter : la question de l'art suisse et de son identité. Ritter ne croit pas à l'idée qu'en ont alors certains critiques. De manière explicite, son rejet de l'œuvre de Ferdinand Hodler s'intensifie avec les discours partisans faisant de lui le chantre de l'art national autour de 1900. L'écrivain Gonzague de Reynold, qui partage quelques sympathies avec son homologue, tout en défendant l'idée de l'helvétisme (la différence dans l'unité), écrivait dans La Voile latine en 1905 :

\begin{abstract}
William Ritter est compliqué, ou plutôt s'est compliqué lui-même [...]. Il a entendu trop de musique, contemplé trop de tableaux [...]. Il connaît trop de langues, ayant vécu en trop de pays opposés : de Neuchâtel à Fribourg, d'Autriche en Roumanie, de Munich à Prague, d'Italie en Allemagne [...]. Les Suisses l'ont brouillé avec la Suisse ; Neuchâtel l'a exaspéré [...]. À peine épargne-t-il Fribourg, parce que Fribourg est une ville patricienne, catholique et souabe, presque slave grâce à ses vitraux de Mehoffer.
\end{abstract}

C'est dans cette même revue romande (en quête d'une identité «latine») que Ritter répondait à une enquête sur la littérature suisse en qualifiant « de "suissarde" cette production toute spéciale à nos cantons protestants qui est la plus écœurante potion que je connaisse, un vomitif sucré » (Ritter I906). Ce qui ne l'empêche pas, dans un même jet de plume, de déclarer la guerre «à la peinture patriotique », et d'affirmer : "Je crois en revanche de toutes mes forces à un art suisse en dehors même des artistes suisses parfois : et de citer les Neuchâtelois Buchser, Boecklin, Sandreuter, Welti, Grasset et... Holbein. 
La relation entre Bille et Ritter repose ainsi sur plusieurs affinités ou homologies, principalement sur un individualisme bien trempé que l'artiste avoue apprécier chez le critique, alors même que ce dernier écrit des choses qui lui déplaisent. Ils partagent tous deux un fantasme féodal ou élitiste quelque peu paradoxal : chez Ritter, cette fiction vit à travers l'héritage de son père, sa collection d'art européen, ses fréquentations mondaines et ses discours marqués par le goût de l'exception et de la différence. Chez Bille, ce fantasme est affiché dans une posture aristocratique, fondée sur une fortune acquise par mariage, sur un mode de vie et un pseudo-château sierrois, en décalage par rapport à son positionnement socialiste.

Les amours et les haines artistiques de Ritter évoluent dans un champ en pleine mutation après 1900 , qui l'incite à se déplacer sans cesse, quitte à contredire ses déclarations antérieures, dans esprit qui combine soucis de distinction et désir de provocation. Son discours sur Holbein en 1919 participe de ce projet. Ritter utilise l'artiste bâlois à rebrousse-poil des jugements de ses contemporains à la fois pour contrer l'idée de "suissitude » et pour dévoyer le discours socialisant de Bille, au nom de son indépendance de critique. Un art de la contredanse, ou, pour reprendre des métaphores musicales, un art de la fugue ou du contrepoint : telle est la partition critique que William Ritter n'a cessé de jouer.

\section{Bibliographie}

Bille, Edmond, Le Carquois vide. Souvenirs d'un arbalétrier 19I4-I9I8, Neuchâtel, La Baconnière, 1939.

Correspondance : Edmond Bille, ALS, Fonds William Ritter.

Journal intime, voir : Ritter, William, « Un journal de toute une vie », ALS, Fonds William Ritter.

Kaenel, Philippe, «Philippe Godet (I850-1922) : le critique d'art critiqué », in Critiques d'art de Suisse romande: de Töpffer à Budry, éd. Philippe Junod \& Philippe Kaenel, Payot, Lausanne 1993, pp. I4I-I85 (Études et documents littéraires).

—. «William Ritter : un critique cosmopolite, böcklinien et anti hodlérien », Revue suisse d'histoire, vol. 48, $\mathrm{n}^{\circ}$ I, I998, pp. 73-98.

—. « La danse macabre de l'ouvrier et du soldat. Edmond Bille face à la Première Guerre mondiale ", Cahiers d'histoire du mouvement ouvrier, 2003, pp. 45-54.

Martinet, Catherine, Edmond Bille (1878-1959). Une danse macabre, Université de Lausanne, 1982 [mémoire de licence].

Reynold, Gonzague de, "Bibliographie : William Ritter », La Voile latine, avril I905, pp. I90-I9I. 
Ritter, William, « Documents : Enquête sur l'art et la littérature suisses », La Voile latine, hiver 1906, pp. 96-98.

- « Correspondance de Suisse. La Société des peintres, sculpteurs et architectes suisses, sa première exposition à Bâle, sa participation à la neuvième exposition internationale de Munich », Gazette des beaux-arts, vol. I, I906, p. 262.

—. «Deux peintres du Valais : Edmond Bille et Raphy Dallèves », Schweizerland, $4^{\text {ème }}$ année, II/I2, août-septembre, Zurich, Coire, [I9I8], pp. 570-572.

—. "Mes relations avec les artistes suisses. XIX. Le Valais », ALS, Fonds William Ritter, carton 28 A-3-b.

—. «Zwei Maler des Wallis », Neue Zürcher Zeitung, 25 mars I9I8.

-. Le Valais et ses peintres : Blanche Berthoud, Edmond Bille... Édouard Vallet, Grande salle de l'Hôtel des Postes, La Chaux-de-Fonds, I2 septembre - 9 octobre I9I8, Salon français Henri Brendlé, cat expo, [s.l.] : [s.é.], [I9I8].

Ritter, William, [préface], dans Edmond Bille, Une Danse macabre, Lausanne, Spes, I9I9.

Traz, Robert de, « Chronique », La Semaine littéraire, 26 avril ı9ı9, p. I9I.

\section{Publications de William Ritter sur Edmond Bille}

Ritter, William, « Correspondance de Suisse. La Société des peintres, sculpteurs et architectes suisses, sa première exposition à Bâle, sa participation à la neuvième exposition internationale de Munich ", Gazette des beaux-arts, vol. I, I906, p. 262.

-. « La X esposizione internazionale di Monaco», Emporium, n I79, novembre I909, Bergame : Inviare Cartolina-Vglia all'Istituto Italiano d'Arti Grafiche, pp. 323-342.

—. "Les artistes neuchâtelois à l'Exposition intern. de Munich », La Suisse libérale, [ $\mathrm{I}^{\mathrm{e} r \mathrm{e}}$ partie] 23.7.1909, [ $2^{\mathrm{e}}$ partie] 26.7.1909.

—. «Deux peintres du Valais : Edmond Bille et Raphy Dallèves », Schweizerland, 4 année, II/I2, août-septembre, Zurich, Coire, [I9I8], pp. 570- 572.

-. «Zwei Maler des Wallis », Neue Zürcher Zeitung, 25 mars I9I8.

—. «Les Deux Valais », Feuille d'Avis de La Chaux-de-Fonds, 30.3.I9I8, [p.2].

—. «L'exposition Bille / Dallèves à Zurich », L’Aube, I.4.I9I8, pp. 2I2-2I3.

- « À l'Exposition valaisanne II », Feuille d'Avis de La Chaux-de-Fonds, 28.9.1918.

-. "À l'Exposition valaisanne V », La Petite Feuille, supplément du dimanche de la Feuille d'Avis [de La Chaux-de-Fonds], 5.Io.I9I8, n 37, [s. p.].

—. « Edmond Bille », O mein Heimatland, Bern, G. Grunau, Zurich, Rascher [?], Genève : Burkhardt, I919, pp. I34-I47. 
-. Le Valais et ses peintres : Blanche Berthoud, Edmond Bille... Édouard Vallet, Grande salle de l'Hôtel des Postes, La Chaux-de-Fonds, I2 septembre - 9 octobre I9I8, Salon français Henri Brendlé, cat expo, [s.l.] : [s.é.], [I9I8].

-. [préface], dans Edmond Bille, Une Danse macabre, Lausanne, Spes, I919. 